S81

FUNCTIONAL CAPACITY, PERIPHERAL MUSCLE STRENGTH, AND QUALITY OF LIFE FOLLOWING INTERVAL VERSUS CONTINUOUS REHABILITATIVE EXERCISE TRAINING IN CYSTIC FIBROSIS

${ }^{1} \mathrm{G}$ Kaltsakas, ${ }^{2} \mathrm{~N}$ Anastasopoulos, ${ }^{3} \mathrm{~N}$ Chynkiamis, ${ }^{4} \mathrm{P}$ Zeliou, ${ }^{4} \mathrm{~V}$ Karapatoucha, ${ }^{4} \mathrm{~K}$ Kotsifas, ${ }^{4} \mathrm{~F}$ Diamantea, ${ }^{4} \mathrm{I}$ Iglezos, ${ }^{1} \mathrm{NG}$ Koulouris, ${ }^{3}$ I Vogiatzis. ${ }^{1}$ 1st Respiratory Medicine Department, "Sotiria" Hospital for Diseases of the Chest, National and Kapodistrian University of Athens, Athens, Greece; ${ }^{2}$ Faculty of Physical Education and Sport Sciences, National and Kapodistrian University of Athens, Athens, Greece; ${ }^{3}$ Faculty of Health and Life Sciences, Department of Sport, Exercise and Rehabilitation, Northumbria University Newcastle, Newcastle Upon Tyne, UK; ${ }^{4}$ Adult Cystic Fibrosis Unit, "Sismanogleion" General Hospital of Attica, Athens, Greece

\subsection{6/thoraxjnl-2017-210983.87}

The effects of pulmonary rehabilitation in cystic fibrosis are well documented, but the effectiveness of interval exercise training remains unexplored. The aim of this study was to investigate whether interval exercise (IE) could be as beneficial as continuous exercise (CLE) in terms of improvement in functional capacity, peripheral muscle strength, and quality of life. We studied 24 Caucasian, ambulatory, adult, cystic fibrosis patients. Patients underwent a structured, outpatient, hospitalbased pulmonary rehabilitation program for 12 weeks. Patients were randomised either to $30 \mathrm{~min}$ high-intensity IE $(100 \%$ WRmax for $30 \mathrm{~s}$ alternated with $40 \%$ WRmax for $30 \mathrm{~s}$; $\mathrm{n}=12)$ or $30 \mathrm{~min}$ moderate intensity CLE (70\% WRmax; $\mathrm{n}=12$ ). Interventions were balanced to provide the same overall training work load. Assessment was performed at baseline and following completion of the rehabilitation program. Functional capacity was assessed by the 6MWT, peripheral muscle strength was measured by the quadriceps isometric force and the Cystic Fibrosis Questionnaire-Revised (CFQ-R) was used to assess patient reported outcomes. The 6MWT was significantly improved equally in the IE Group (by $45 \mathrm{~m}$; pre: 538 \pm 70 , post: $583 \pm 83 \mathrm{~m} ; \mathrm{p}<0.001$ ) and in CLE Group (by $48 \mathrm{~m}$; pre: $516 \pm 57$, post: $564 \pm 55 \mathrm{~m} ; \mathrm{p}=0.001)$. Improvement in quadriceps muscle strength was significantly greater in the IE group (pre: $37.9 \pm 13.1$, post: $45.2 \pm 14.2 \mathrm{Kg} ; \mathrm{p}=0.024$ ) compared to CLE (pre: $40.0 \pm 12.2$, post: $45.4 \pm 9.3 \mathrm{Kg}$; $\mathrm{p}=0.072$ ). The CFQ-R was improved in both groups for the domains of "physical functioning" (IE Group pre: 52.8 \pm 14.5 , post: $64.6 \pm 11.9$; CLE Group pre: $60.4 \pm 11.2$, post: 67.4 $\pm 13.1 ; \mathrm{p}=0.034$ ) and "body image" (IE Group pre: 81.5 \pm 8.5 , post: $88.1 \pm 7.3$; CLE Group pre: $74.1 \pm 10.7$, post: $80.7 \pm 9.7 ; \mathrm{p}=0.046)$. Average training arterial oxygen saturation and Borg dyspnoea scores during exercise training indicated that the IE Group compared to the CLE Group exhibited higher oxygen saturation $(94 \pm 1$ vs. $91 \% \pm 1 \%$; $\mathrm{p}=0.002)$ and lower intensity of dyspnoea (3.8 \pm 0.7 vs. 5.9 $\pm 0.8 ; \mathrm{p}<0.001)$.

In conclusion, within the pulmonary rehabilitation setting, IE is equally effective to CLE in improving functional capacity and aspects of quality of life, but is superior to CLE in improving peripheral muscle strength. Furthermore, it can be applied to CF patients with lower dyspnoea sensations and lower arterial desaturation, thus qualifying as a safer alternative training strategy.

\section{S82 DAILY STEPPING DOES NOT RECOVER AS AN INPATIENT: STANDARDISING THE MEASUREMENT OF PHYSICAL ACTIVITY DURING HOSPITALISATION FOR RESPIRATORY DISEASE}

${ }^{1} \mathrm{MW}$ Orme, ${ }^{1} \mathrm{TC}$ Harvey-Dunstan, ${ }^{2}$ Boral, ${ }^{1} \mathrm{EJL}$ Chaplin, ${ }^{2} \mathrm{~S}$ Fayyaz Hussain, ${ }^{1} \mathrm{MC}$ Steiner, ${ }^{1} \mathrm{SJ}$ Singh, ${ }^{1} \mathrm{NJ}$ Greening. ${ }^{1}$ Centre for Exercise and Rehabilitation Science, NIHR Leicester Biomedical Research Centre - Respiratory, Leicester, UK; ${ }^{2}$ Respiratory Department, Kettering General Hospital NHS Foundation Trust, Kettering, UK

\subsection{6/thoraxjnl-2017-210983.88}

Introduction and Objectives Hospitalisation for an exacerbation of chronic respiratory disease has a major impact on physical activity (PA). However, criteria to derive reliable inpatient PA data do not exist and current recommendations are unlikely to account for variations in length of hospital stay (LOHS) and the hospital environment. The aims were to identify the minimum wear time and number of days required to obtain reliable inpatient PA data; to use these criteria to determine how PA changes during recovery as an inpatient; and to compare PA across patients stratified by LOHS.

Methods 259 individuals hospitalised with an exacerbation of chronic respiratory disease were recruited as part of an early rehabilitation trial previously reported (Greening et al, BMJ 2014). Participants (mean (SD) age $70.0 \pm 9.7$ years, $58.3 \%$ female) wore a physical activity monitor (SenseWear) during their stay. Daily step count and walking time during waking hours was analysed. Inpatient PA was assessed across a range of minimum wear time criteria ( $\geq 1-12$ hours). Repeated measures analysis of covariance was used to compare between days and between times of day. Single-day intraclass correlation coefficients (ICCs) were calculated across the range of wear time criteria. The minimum number of days required to obtain an ICC $\geq 0.80$ was estimated using the SpearmanBrown prophecy formula.

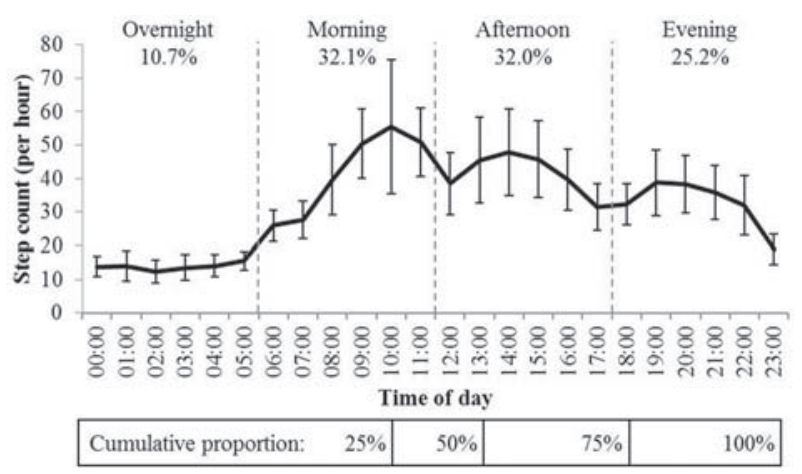

Abstract S82 Figure 1 Step count per hour as a proportion of total daily step count across an average 24 hour period. Data are reported as mean $(95 \% \mathrm{Cl})$. 